\title{
AC-Conductivity of Thin Polycrystalline Tin Dioxide Films
}

\author{
V.K. Ksenevich ${ }^{a, *}$, N.I. GorbachuK ${ }^{a}$, T.A. Dauzhenka ${ }^{a, b}$, I.A. Bashmakov ${ }^{c}$, \\ N.A. POKLONSKI ${ }^{a}$ AND A.D. WIECK ${ }^{d}$ \\ ${ }^{a}$ Department of Physics, Belarus State University, Nezalezhnastsi ave. 4, 220030 Minsk, Belarus \\ ${ }^{b}$ CNRS; LNCMI; 143 Av. de Rangueil, F-31400 Toulouse, France \\ ${ }^{c}$ Research Institute for Chemical Problems, Belarus State University, Leningradskaja str., 14, 220050 Minsk, Belarus \\ ${ }^{d}$ Department of Physics and Astronomy, Bochum Ruhr-University, Universitaetstr., 150, 44780 Bochum, Germany \\ The electrical properties of polycrystalline tin dioxide films were investigated by impedance spectroscopy in \\ the frequency range $100 \mathrm{~Hz}-1 \mathrm{MHz}$ at temperatures $4.2 \mathrm{~K}, 77 \mathrm{~K}$ and $300 \mathrm{~K}$. Analysis of the experimental data \\ by means of complex nonlinear least squares method made it possible to divide the contributions of grain bulk \\ and grain boundaries to the conductivity. It was found that at room temperature charge transport processes are \\ mainly determined by the grain volume while at the low temperatures contribution from the grain boundaries to \\ the impedance of the system prevails.
}

PACS: 73.61.Le

\section{Introduction}

Tin dioxide nanocrystalline structures are used for number of optoelectronic applications due to their high conductivity and high transparency in the visible range of electromagnetic spectra $[1,2]$. Investigation of influence of stoichiometry, doping, grain sizes etc. on the conductivity of tin dioxide is very important. Measurements of the complex impedance are one of the more reliable methods in order to divide contribution from the grain boundaries and grain volume in the total conductivity of polycrystalline materials [3].

\section{Experimental results}

Polycrystalline tin dioxide films were fabricated using gas-transport chemical reaction on the previously synthesized metal-polymer precursor [4]. The grain size of the samples was in the range $20-100 \mathrm{~nm}$ according to scanning electron microscopy and transmission electron microscopy images. Impedance spectroscopy measurements in the frequency range from $100 \mathrm{~Hz}$ to $1 \mathrm{MHz}$ and in the temperature range $4.2-300 \mathrm{~K}$ were used for the electrical characterization of the samples. LCR meter Agilent 4284A was used for the measurements of frequency dependences of real and imaginary components of the impedance of tin dioxide films. Amplitude of the sinusoidal signal was $25 \mathrm{mV}$. DC bias voltage in the range $0-3 \mathrm{~V}$ was simultaneously applied to samples. Dependences of AC-conductivity on the applied bias voltage were measured in the temperature range $4.2-300 \mathrm{~K}$ as well.

* corresponding author; e-mail: ksenevich@bsu.by

\section{Discussion}

Frequency dependences of the real and imaginary components of the impedance of polycrystalline tin dioxide films $Z^{\prime}(f)$ and $Z^{\prime \prime}(f)$, measured at temperatures $4.2 \mathrm{~K}, 77 \mathrm{~K}$ and $300 \mathrm{~K}$, are shown in Figs. 1 and 2, respectively. The points correspond to the experimental data while solid lines represent the results of the calculations by means of complex nonlinear least-squares method (CNLS) [5]. It was found that the impedance of the samples does not depend on the applied bias voltage at $77 \mathrm{~K}$ and $300 \mathrm{~K}$ and decreases with the increase of bias voltage at $T=4.2 \mathrm{~K} . Z^{\prime}(f)$ dependences measured at $U_{\mathrm{b}}=0 \mathrm{~V}$ and $U_{\mathrm{b}}=3 \mathrm{~V}$ are shown in Fig. 1 . As one can see from Fig. 2, maximum observed on the $Z^{\prime \prime}(f)$ dependences shifts to the high-frequency region with the increase of the temperature and bias voltage (at $T=4.2 \mathrm{~K}$ ). Nonlinear dependences of the impedance on the applied DC bias voltage were observed at $T=4.2 \mathrm{~K}$.

Best fitting results were obtained by simulation of the experimental data with an equivalent scheme shown in the inset to Fig. 1. Resistors $R_{\mathrm{g}}$ and $R_{\mathrm{gb}}$ in this scheme simulate resistance of the grain volume and grains boundaries, respectively. $C_{\mathrm{p}}$ is the parasitic capacitance of the bonding wires. Constant phase element (CPE) used in an equivalent scheme takes into account spread in values of the resistance and capacitance of the grain boundaries. The admittance of the CPE is defined as [5]:

$$
\begin{aligned}
& Y_{\mathrm{CPE}}=A_{0}(\mathrm{i} \omega)^{\alpha} \\
& \quad=A_{0} \omega^{\alpha}[\cos ((\pi / 2) \alpha)+\mathrm{i} \sin ((\pi / 2) \alpha)],
\end{aligned}
$$

where $A_{0}$ - coefficient with the dimensionality depending on the $\alpha$ value. In the case $\alpha=1, A_{0}$ has the dimensionality of capacitance while in the case $\alpha=0, A_{0}$ has the dimensionality of resistance. In the intermediate case dimensionality of $A_{0}$ can be considered as $\Omega^{-1} \mathrm{~s}^{\alpha}[6]$. 


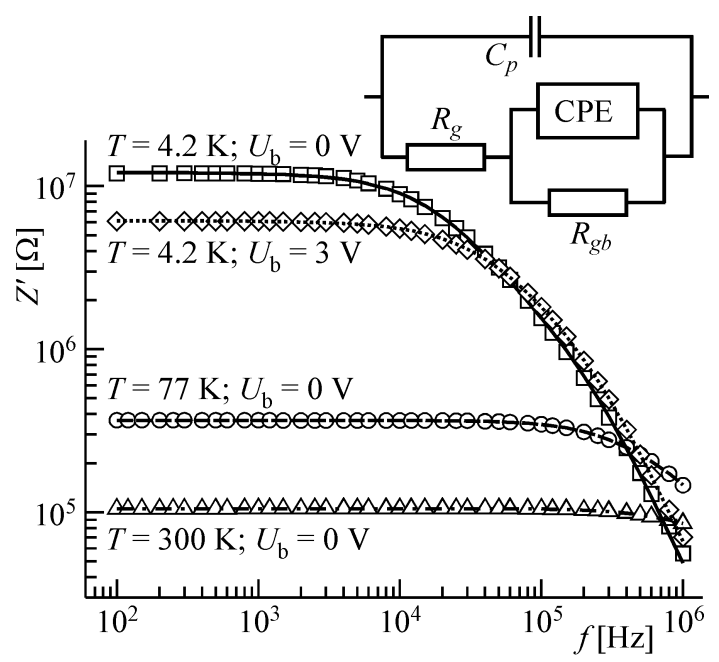

Fig. 1. Frequency dependence of the real component of the impedance of polycrystalline tin dioxide films measured at different temperatures and applied DC bias voltage. Lines show the approximation of the experimental data by an equivalent circuit, shown in the inset.

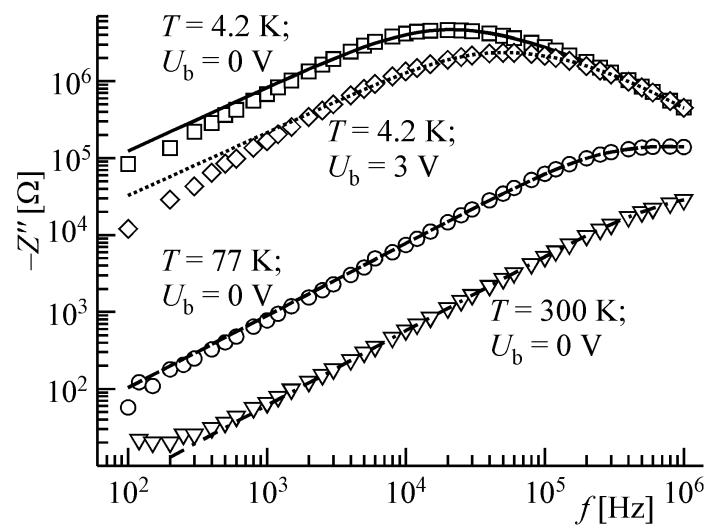

Fig. 2. Frequency dependence of the imaginary component of the impedance of polycrystalline tin dioxide films measured at different temperatures and applied DC bias voltage. Lines show the approximation of the experimental data by an equivalent circuit.

As one can see from Figs. 1 and 2, frequency dependences of the impedance can be well fitted at $f>300 \mathrm{~Hz}$ by an equivalent scheme shown in the inset to Fig. 1. According to approximation results at room temperature resistance of grain volume is about 4 times higher than the resistance of the grain boundaries $\left(R_{\mathrm{g}}=8.5 \times 10^{4} \Omega\right.$ vs. $R_{\mathrm{gb}}=2.0 \times 10^{4} \Omega$ ).

As the temperature decreases, contribution to the impedance of the samples from the grain boundaries is rising $\left(R_{\mathrm{g}}=2.5 \times 10^{5} \Omega, R_{\mathrm{gb}}=1.2 \times 10^{5} \Omega\right.$ at $\left.T=77 \mathrm{~K}\right)$. At $T=4.2 \mathrm{~K}$ the resistance of the grain boundaries exceeds the resistance of grain volume $\left(R_{\mathrm{g}}=3.9 \times 10^{6} \Omega\right.$ vs. $R_{\mathrm{gb}}=8.2 \times 10^{6} \Omega$ ). Thus, impedance and nonlinearity of the conductivity of thin polycrystalline tin dioxide films at $T=4.2 \mathrm{~K}$ are determined mainly by the grain boundaries. Temperature rise is accompanied by the essential increase of their conductivity. As a result, nonlinear electrical properties were not observed at the liquid nitrogen and room temperatures. Impedance of the samples in the high temperature range is determined mainly by the resistance of the volume of tin dioxide grains.

\section{Conclusion}

Analysis of the complex impedance of polycrystalline tin dioxide films, measured at different temperatures and different applied DC bias voltage was done. It was found that at room temperature electrical properties are determined by the contribution to the impedance both from the grain volume and grain boundary. As the temperature decreases, contribution from the grain boundaries to the impedance of the system starts to prevail.

\section{Acknowledgments}

The work was supported by the Foundation of Basic Research of Republic of Belarus (grant No. F09F-008) and National Research Program "Nanomaterials and Nanotechnologies" (grant No. 5.17(3)). V.K. and N.G. would like to acknowledge DAAD for financial support and Prof. A.D. Wieck for hospitality.

\section{References}

[1] S. Mathur, S. Barth, H. Shen, J.C. Pyun, U. Werner, Small 1, 713 (2005)

[2] S. Ferrere, A. Zaban, B.A. Gsegg, J. Phys. Chem. B 101, 4490 (1997).

[3] N. Bonanos, B.C.H. Steele, E.P. Butler, in: Impedance Spectroscopy: Theory, Experiment, and Applications, Eds. E. Barsoukov, J.R. Macdonald, Wiley, Hoboken, NJ 2005, p. 205.

[4] I.A. Bashmakov, T.A. Dauzhenka, V.K. Ksenevich, in: Proc. Int. Scientific Conf. "Actual Problems of Solid State Physics", Vol. 2, Ed. N.M. Olekhnovich, Varaksin, Minsk 2009, p. 43.

[5] I.D. Raistrick, D.R. Franceschetti, J.R. Macdonald, in Ref. [3], p. 27.

[6] M. Sluyters-Rehbach, Pure Appl. Chem. 66, 1831 (1994). 